\title{
SLANT CURVES AND PARTICLES IN THREE-DIMENSIONAL WARPED PRODUCTS AND THEIR LANCRET INVARIANTS
}

\author{
CONSTANTIN CĂLIN ${ }^{\bowtie}$ and MIRCEA CRASMAREANU
}

(Received 16 July 2012; accepted 9 August 2012; first published online 19 October 2012)

\begin{abstract}
Slant curves are introduced in three-dimensional warped products with Euclidean factors. These curves are characterised by the scalar product between the normal at the curve and the vertical vector field, and an important feature is that the case of constant Frenet curvatures implies a proper mean curvature vector field. A Lancret invariant is obtained and the Legendre curves are analysed as a particular case. An example of a slant curve is given for the exponential warping function; our example illustrates a proper (that is, not reducible to the two-dimensional) case of the Lancret theorem of three-dimensional hyperbolic geometry. We point out an eventuality relationship with the geometry of relativistic models.
\end{abstract}

2010 Mathematics subject classification: primary 53B25; secondary 53A55, 53C25, 53C40.

Keywords and phrases: warped product, slant curve, Legendre curve, Lancret invariant, Frenet frame.

\section{Introduction}

Recall that in the Euclidean differential geometry of space curves an important class of examples is provided by curves of constant slope. The name is justified by the fact that for such a curve $\gamma$ its tangent vector field has a constant angle $\theta$ with a fixed direction called the axis of $\gamma$. Cylindrical helices is another well-known name for this class since there exists a cylinder on which $\gamma$ moves in such a way as to cut each ruling at a constant angle. The Bertrant-Lancret-de Saint Venant theorem [2] is the technical characterisation: the curve $\gamma$ in $\mathbb{E}^{3}$ is of constant slope if and only if the ratio of torsion and curvature is constant. Then for a cylindrical helix we have the Lancret invariant:

$$
\operatorname{Lancret}(\gamma)=\frac{\cos \theta}{|\sin \theta|}=\frac{\tau}{k}
$$

An extension of this class to the almost contact metric geometry was introduced in [10] under the name of slant curve: the axis of $\gamma$ is now the Reeb vector field. In

The second author has been supported by the Romanian National Authority for Scientific Research, CNCS UEFISCDI, project number PN-II-ID-PCE-2012-4-0131.

(C) 2012 Australian Mathematical Publishing Association Inc. 0004-9727/2012 \$16.00 
the particular case $\theta=\pi / 2$ (or $\theta=3 \pi / 2$ ) we recover the Legendre curves of [1]. Slant curves in three-dimensional Sasakian manifolds have, after [10, Theorem 3.1, p. 362],

$$
\text { Lancret }(\gamma)=\frac{\cos \theta}{|\sin \theta|}=\frac{\tau \pm 1}{k} \text {. }
$$

The literature on Legendre curves comes under several titles [3, 6, 9, 15, 17, 19-22] while slant curves have hitherto been studied only in Sasakian geometry [10], contact pseudo-Hermitian geometry [11], normal almost contact geometry [7], $f$-Kenmotsu geometry [8] and, under the name of generalised helices, in Lie groups with biinvariant metric in [12]. The purpose of this paper is to begin a study of slant curves in another important class of manifolds introduced by Bishop and O'Neill [4] in order to construct a large class of manifolds with negative curvature.

Our work is structured as follows. Section 2 is a very brief review of (threedimensional) warped products and Frenet curves in general Riemannian geometry. Section 3 is devoted to the study of slant (particularly Legendre) curves in this generalised framework of warped products with Euclidean factors. So we obtain a characterisation of slant curves similar to [10, Proposition 3.1, p. 361] and an a priori estimate (inequality) in terms of the slant angle, the logarithm of the warping function and the curvature of the curve. Two cases of equality for this inequality are analysed.

Section 4 is the main part of this paper and begins with a classification of all slant curves. Based on the general expression of these curves, their curvature and torsion are computed and a Lancret invariant is naturally associated. Our Lancret expression is more complicated, involving, in addition to the fraction of curvature and torsion, a square root and the direct trigonometric functions in the slant angle $\theta$. The particular case of Legendre curves is discussed for curvature and torsion. This section ends with an important property, namely that these curves with constant curvature and torsion, that is, general helices, have proper mean curvature vector field or, more precisely, the normal vector field is an eigenvalue of the Laplacian.

Section 5 is devoted to examples. After a short look at the Euclidean case considered as a trivial warped product, an example of a slant curve is studied for exponential warped product manifolds. Let us point out that the case of a warping function $f$ of exponential type appears as an extremal case since from relation (3.3) of Section 3 we obtain that $f$ can have at most exponential growth.

Section 6 discuss the possible applications for the trajectories of particles of slant type in relativistic models; two open problems are naturally raised, with respect to both dynamical and geometrical aspects of these particles.

\section{Warped products and Frenet curves}

Let $B$ and $N$ be two smooth manifolds endowed with the Riemannian metrics $g_{B}$ and $g_{N}$ and with dimension $b$ and $n$ respectively. Let $f: B \rightarrow \mathbb{R}_{+}^{*}$ be a smooth and strictly positive function. The warped product of $B$ and $F$ with warping function $f$ is the Riemannian manifold

$$
B \times_{f} N=\left(M_{b+n}, g\right)=\left(B \times N, g_{B}+f^{2} g_{N}\right)
$$


where in the right-hand side the function $f$ is in fact $f \circ \pi$ with $\pi: B \times N \rightarrow B$ the projection on the first factor.

In the following we restrict ourselves to the case where $B=I$, an open real interval with the Euclidean metric, and $N=\mathbb{E}^{2}$ is the Euclidean plane. We use the classical coordinates $(x, y)$ on $\mathbb{E}^{2}$ and $z$ on $I$; therefore the main vector field considered below on $\left(M_{3}, g\right)=I \times_{f} \mathbb{E}^{2}$, namely $\partial / \partial z=\partial_{z}$, will be called the vertical vector field. The warping function is then $f=f(z)$, and for further use we consider the function $F: B \rightarrow \mathbb{R}$, where

$$
F=\log f .
$$

Denote by $\nabla$ the Levi-Civita connection of $\left(M_{3}, g\right)$ and by $U, V$ lifts to $M$ of vector fields tangent to $\mathbb{E}^{2}$. Then

$$
\begin{aligned}
& \nabla_{U} V=D_{U} V-F^{\prime} g(U, V) \partial_{z}, \\
& \nabla_{U} \partial_{z}=\nabla_{\partial_{z}} U=F^{\prime} U, \\
& \nabla_{\partial_{z}} \partial_{z}=0
\end{aligned}
$$

where $D$ is the covariant derivative on $\mathbb{E}^{2}$ and prime denotes the derivative with respect to the variable $z$. Also, denote by $\eta=d z$ the dual of the vertical vector field.

In the last part of this section we recall the notion of a Frenet curve in an $\mathrm{m}$ dimensional Riemannian manifold $\left(M_{m}, g\right)$ following [5, p. 164]. Let $r$ be an integer with $1 \leq r \leq m$. The curve $\gamma: J \subseteq \mathbb{R} \rightarrow M$ parametrised by the arc length $s$ is called an $r$-Frenet curve on $M$ if there exist $r$ orthonormal vector fields $\left(E_{1}=\gamma^{\prime}, \ldots, E_{r}\right)$ along $\gamma$ such that there exist positive smooth functions $k_{1}, \ldots, k_{r-1}$ of $s$ with

$$
\left\{\begin{array}{l}
\nabla_{\gamma^{\prime}} E_{1}=k_{1} E_{2}, \\
\nabla_{\gamma^{\prime}} E_{2}=-k_{1} E_{1}+k_{2} E_{3}, \\
\ldots, \\
\nabla_{\gamma^{\prime}} E_{r}=-k_{r-1} E_{r-1} .
\end{array}\right.
$$

The function $k_{j}$ is called the jth curvature of $\gamma$ while $\gamma$ is:

(1) a geodesic if $r=1$, so we get the well-known equation $\nabla_{\gamma^{\prime}} \gamma^{\prime}=0$;

(2) a circle if $r=2$ and $k_{1}$ is a constant, so we have $\nabla_{\gamma^{\prime}} E_{1}=k_{1} E_{2}$ together with $\nabla_{\gamma^{\prime}} E_{2}=-k_{1} E_{1}$;

(3) a helix of order $r$ if $k_{1}, \ldots, k_{r-1}$ are constants.

The Frenet curve $\gamma$ is called nongeodesic if $k_{1}>0$ everywhere on $I$.

\section{Slant and Legendre curves in three-dimensional warped products}

Fix a 3-Frenet curve $\gamma$ in $M_{3}=I \times_{f} \mathbb{E}^{2}$ (so $r=m=3$ ), for which we denote the Frenet frame as usual $\left(T=\gamma^{\prime}, N, B\right)$, and the Frenet equations (2.2) are

$$
\left\{\begin{array}{l}
\nabla_{T} T=k N, \\
\nabla_{T} N=-k T+\tau B, \\
\nabla_{T} B=-\tau N .
\end{array}\right.
$$


Definition 3.1. (i) The structural angle of $\gamma$ is the function $\theta: J \rightarrow[0,2 \pi)$ given by

$$
\cos \theta(s)=g\left(T(s), \partial_{z}\right)=\eta(T(s)) .
$$

(ii) We call $\gamma$ a slant curve (or more precisely a $\theta$-slant curve) if $\theta$ is a constant function [10, p. 361]. In the particular case where $\theta \equiv \pi / 2$ (or $3 \pi / 2$ ), $\gamma$ is called a Legendre curve [1].

Example 3.2. (i) The integral curves of the vertical vector field $\partial_{z}$ are slant curves with $\theta \equiv 0$. These curves are $\gamma(s)=\left(x_{0}, y_{0}, s+z_{0}\right)$ with $s \in J=\mathbb{R}$ and $\gamma(0)=$ $\left(x_{0}, y_{0}, z_{0}\right)$ the initial point. From (2.1c) these curves are geodesics for the warped metric $g$.

(ii) The tangent vector field of a Legendre curve belongs to the contact distribution $\mathcal{D}:=\operatorname{Ker} \eta$. A remarkable fact of the warped geometry $I \times_{f} N$ in any dimension is that $\mathcal{D}$ defines a Riemannian foliation since it is integrable from $d \eta=0$.

In the following we suppose that $\gamma$ is nongeodesic, that is, $k>0$ and then $\gamma$ cannot be an integral curve of $\xi$ which means that $\theta \neq 0, \pi$. A first main result is the following characterisation of slant curves and an a priori estimate.

Proposition 3.3. The Frenet curve $\gamma$ is a $\theta$-slant curve if and only if along $\gamma$ the following relation holds:

$$
\eta(N)=-\frac{F^{\prime}}{k} \sin ^{2} \theta .
$$

Then a necessary condition for $\gamma$ to be $\theta$-slant is

$$
|\sin \theta| \leq \min \left\{1, \frac{k}{\left|F^{\prime}\right|}\right\}
$$

if $f$ is not a constant function.

Proof. Suppose that $T(s)=\left(T_{1}(s), T_{2}(s), T_{3}(s)=\cos \theta\right)$. From (2.1b)-(2.1c),

$$
\nabla_{T} \partial_{z}=\nabla_{\left(T_{1}, T_{2}, 0\right)} \partial_{z}=F^{\prime}\left(T_{1}, T_{2}, 0\right)=F^{\prime}\left(T-\cos \theta \partial_{z}\right) .
$$

Let us covariantly derive the relation (3.1) along $\gamma$ :

$$
0=-\theta^{\prime} \sin \theta=g\left(k N, \partial_{z}\right)+g\left(T, F^{\prime}\left(T-\cos \theta \partial_{z}\right)\right)=k \eta(N)+F^{\prime} \sin ^{2} \theta,
$$

which yields (3.2). The expression of $\partial_{z}$ in the Frenet frame is

$$
\partial_{z}=\cos \theta T+\left(-\frac{F^{\prime}}{k} \sin ^{2} \theta\right) N+\eta(B) B,
$$

and since $\partial_{z}$ is a unitary vector field we get from this decomposition that

$$
1=\cos ^{2} \theta+\frac{F^{\prime 2} \sin ^{4} \theta}{k^{2}}+\eta(B)^{2},
$$

and then $\eta(B)$ there exists only if the condition $\left|F^{\prime} \sin \theta\right| \leq k$ holds, which means that (3.3) is satisfied. 
REMARKs 3.4. (i) The decomposition of $\partial_{z}$ in the Frenet frame of a slant curve is

$$
\partial_{z}=\cos \theta T+\left(-\frac{F^{\prime}}{k} \sin ^{2} \theta\right) N+\left(\frac{|\sin \theta| \sqrt{k^{2}-F^{\prime 2} \sin ^{2} \theta}}{k}\right) B ;
$$

and then, for a Legendre curve, $\partial_{z}$ is in the plane spanned by $N$ and $B$.

(ii) The equality $|\sin \theta|=k /\left|F^{\prime}\right|$ implies $\eta(B)=0$, and then

$$
\partial_{z}=\cos \theta T \pm|\sin \theta| N .
$$

Applying $\nabla_{T}$ to this equality gives

$$
F^{\prime}(T-\cos \theta(\cos \theta T \pm|\sin \theta| N))=(\cos \theta) k N \pm|\sin \theta|(-k T+\tau B) ;
$$

analysing the coefficient of $B$, we derive that $\tau=0$, and then $\gamma$ can be thought of as a circle. Also, it follows that $F^{\prime}<0$.

(iii) In [16, p. 155] the following notion is introduced: a nongeodesic curve is called a slant helix if the principal normal lines of $\gamma$ make a constant angle with a fixed direction. Therefore, a slant curve with $k$ a nonzero constant in a three-dimensional warped product having $f(z)=\exp (\beta z)$, where $\beta \in \mathbb{R}$ satisfies $|\beta \sin \theta| \leq k$, is a slant helix with $\partial_{z}$ as fixed direction. Let us call this manifold an exponential Euclidean warped product in what follows.

\section{Classification of slant curves, the Lancret invariant and their proper mean curvature field}

In the following theorem we classify all $\theta$-slant curves, inspired by an idea of [8].

Theorem 4.1. Let $\gamma$ be a $\theta$-slant curve in $I \times_{f} \mathbb{E}^{2}$ defined on $J \subseteq \mathbb{R}$. Then $\gamma$ is given by

$$
\gamma(s)=\left(|\sin \theta| \int^{s} \frac{\zeta(t) d t}{f(t \cos \theta)}, s \cos \theta\right)
$$

where $\zeta(s)=(\cos \alpha(s), \sin \alpha(s))$, with $\alpha \in C^{\infty}(J)$, is an arbitrary parametrisation of the unit circle $\mathbb{S}^{1}$.

Proof. As in the proof of Proposition 3.3, from $T(s)=\left(T_{1}(s), T_{2}(s), \cos \theta\right)$ with $\|T\|^{2}=1$ we get

$$
1=f^{2}(s \cos \theta)\left(T_{1}^{2}+T_{2}^{2}\right)+\cos ^{2} \theta
$$

which yields

$$
T_{1}^{2}+T_{2}^{2}=\frac{\sin ^{2} \theta}{f^{2}(s \cos \theta)}
$$

Therefore

$$
T_{1}=\frac{|\sin \theta|}{f(s \cos \theta)} \cos \alpha(s), \quad T_{2}=\frac{|\sin \theta|}{f(s \cos \theta)} \sin \alpha(s)
$$

and we get the desired conclusion. 
Now we are able to compute the Frenet invariants.

THEOREM 4.2. The curvature and the torsion of a $\theta$-slant curve are

$$
\begin{aligned}
& k=|\sin \theta| \sqrt{\left(\alpha^{\prime}\right)^{2}+\left(F^{\prime}\right)^{2}}, \\
& \tau=\left|\alpha^{\prime}\right| \cos \theta-\frac{\left|\alpha^{\prime}\right| F^{\prime \prime} \cos \theta-\operatorname{sgn}\left(\alpha^{\prime}\right) \alpha^{\prime \prime} F^{\prime}}{\left(\alpha^{\prime}\right)^{2}+\left(F^{\prime}\right)^{2}},
\end{aligned}
$$

where $\operatorname{sgn}\left(\alpha^{\prime}\right)=\alpha^{\prime} /\left|\alpha^{\prime}\right|$.

Proof. A long but straightforward computation gives

$\nabla_{T} T=\left(\frac{|\sin \theta|}{f}\left(-\alpha^{\prime} \sin s+F^{\prime} \cos \theta \cos s\right), \frac{|\sin \theta|}{f}\left(\alpha^{\prime} \cos s+F^{\prime} \cos \theta \sin s\right),-F^{\prime} \sin ^{2} \theta\right)$ and from $k^{2}=\left\|\nabla_{T} T\right\|^{2}$ we obtain (4.2a).

For (4.2b) we derive covariantly the relation (3.2),

$$
g\left(-k T+\tau B, \partial_{z}\right)+g\left(N, F^{\prime}\left(T-\cos \theta \partial_{z}\right)\right)=-\frac{d}{d s}\left(\frac{F^{\prime}}{k}\right) \sin ^{2} \theta,
$$

and, using the Frenet equations and (3.2) again,

$$
\tau \eta(B)=k \cos \theta-\frac{\left(F^{\prime}\right)^{2}}{k} \cos \theta \sin ^{2} \theta-\frac{d}{d s}\left(\frac{F^{\prime}}{k}\right) \sin ^{2} \theta .
$$

Using $\eta(B)$ in (3.4) we obtain (4.2b).

Remarks 4.3. (i) Returning to Remarks 3.4, the equality $|\sin \theta|=k /\left|F^{\prime}\right|$ implies that $\alpha^{\prime}=0$, and then $\alpha$ is a constant function $\alpha(s)=\alpha_{0} \in \mathbb{R}$.

(ii) The characterisation (3.2) becomes:

$$
\eta(N)=-\frac{F^{\prime}|\sin \theta|}{\sqrt{\left(\alpha^{\prime}\right)^{2}+\left(F^{\prime}\right)^{2}}} .
$$

Another main result gives a Lancret-type invariant for slant curves.

Proposition 4.4. If $\gamma$ is a nongeodesic $(k>0) \theta$-slant curve then it has the Lancret invariant

$$
\operatorname{Lancret}(\gamma)=\frac{\tau}{k} \frac{\left(\left(\alpha^{\prime}\right)^{2}+\left(F^{\prime}\right)^{2}\right)^{3 / 2}}{\left|\alpha^{\prime}\right|\left(\left(\alpha^{\prime}\right)^{2}+\left(F^{\prime}\right)^{2}-F^{\prime \prime}\right)}+\frac{\operatorname{sgn}\left(\alpha^{\prime}\right) \alpha^{\prime \prime} F^{\prime}}{\left|\alpha^{\prime}\right|\left(\left(\alpha^{\prime}\right)^{2}+\left(F^{\prime}\right)^{2}-F^{\prime \prime}\right)|\sin \theta|} .
$$

Proof. The above expression is $\operatorname{Lancret}(\gamma)=\cos \theta /|\sin \theta|$, and we have the conclusion.

In particular, the Legendre curves have

$$
k=\sqrt{\left(\alpha^{\prime}\right)^{2}+\left(F^{\prime}\right)^{2}}, \quad \tau=\frac{\operatorname{sgn}\left(\alpha^{\prime}\right) \alpha^{\prime \prime} F^{\prime}}{\left(\alpha^{\prime}\right)^{2}+\left(F^{\prime}\right)^{2}},
$$

implying that Legendre curves with $\alpha^{\prime}$ constant (particularly vanishing; see Remark 4.3(i)) can be thought of as circles, since $\tau=0$. 
Now let $h$ be the second fundamental form of $\gamma$ and $H$ its mean curvature field:

$$
H=\operatorname{Tr}(h)=h(T, T)=\nabla_{T} T .
$$

Then the Laplacian of $H$ is

$$
\Delta H=-\nabla_{T} \nabla_{T} \nabla_{T} T
$$

and $\gamma$ is called a curve with proper mean curvature vector field if there exists $\lambda \in C^{\infty}(\gamma)$ such that

$$
\Delta H=\lambda H
$$

and if, in particular, $\lambda=0$ then $\gamma$ is a curve with harmonic mean curvature vector field. The last equation becomes

$$
-\Delta H=\left(-3 k^{\prime} k\right) T+\left(k^{\prime \prime}-k^{3}-k \tau^{2}\right) N+\left(2 k^{\prime} \tau+k \tau^{\prime}\right) B=\lambda(-k N),
$$

which means that $k$ and $\tau$ are constants and, for $k \neq 0$,

$$
\lambda=k^{2}+\tau^{2},
$$

which yields the following result.

Proposition 4.5. A slant curve has a proper mean curvature vector field if and only if the expressions

$$
\left\{\begin{array}{l}
\left(\alpha^{\prime}\right)^{2}+\left(F^{\prime}\right)^{2} \\
\left|\alpha^{\prime}\right| \cos \theta-\frac{F^{\prime \prime}\left(\left(\alpha^{\prime}\right)^{2} \cos \theta+\left(F^{\prime}\right)^{2}\right)}{\left|\alpha^{\prime}\right|\left(\left(\alpha^{\prime}\right)^{2}+\left(F^{\prime}\right)^{2}\right)}
\end{array}\right.
$$

are constants (equivalently it is a helix of order three), and then

$$
\lambda=\left(\left(\alpha^{\prime}\right)^{2}+\left(F^{\prime}\right)^{2}\right) \sin ^{2} \theta+\left(\left|\alpha^{\prime}\right| \cos \theta-\frac{F^{\prime \prime}\left[\left(\alpha^{\prime}\right)^{2} \cos \theta+\left(F^{\prime}\right)^{2}\right]}{\left|\alpha^{\prime}\right|\left[\left(\alpha^{\prime}\right)^{2}+\left(F^{\prime}\right)^{2}\right]}\right)^{2} .
$$

Proof. The constancy of the above expressions means the constancy of the curvature and torsion, while expression (4.6) is the application of formula (4.4) with $k$ and $\tau$ from (4.2a) and (4.2b).

An important observation is that the first condition (4.5) is universal, that is, does not depend on the slant angle $\theta$. Therefore, the same conditions are required for Legendre curves, for which

$$
\lambda=\left(\alpha^{\prime}\right)^{2}+\left(F^{\prime}\right)^{2}+\frac{\left(F^{\prime \prime}\right)^{2}\left(F^{\prime}\right)^{4}}{\left(\alpha^{\prime}\right)^{2}\left(\left(\alpha^{\prime}\right)^{2}+\left(F^{\prime}\right)^{2}\right)} .
$$

\section{Examples}

Example 5.1. The Euclidian space $\mathbb{E}^{3}$ is a trivial warped product, that is, $f \equiv 1$; then $F \equiv 0$. The curve

$$
\gamma(s)=\left(|\sin \theta| \sin s+x_{0},-|\sin \theta| \cos s+y_{0}, s \cos \theta+z_{0}\right)
$$




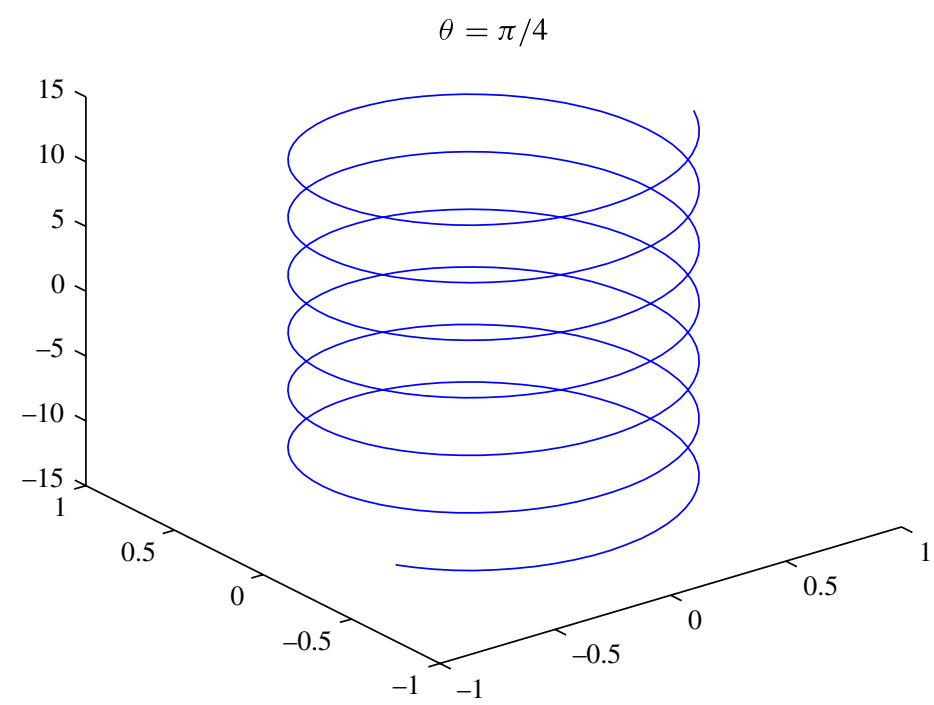

FIgURE 1. The first curve.

is a $\theta$-slant curve (it corresponds to $\alpha(s)=s$ ) and has the Frenet frame

$$
\left\{\begin{array}{l}
T(s)=(|\sin \theta| \cos s,|\sin \theta| \sin s, \cos \theta), \\
N(s)=(-\sin s, \cos s, 0), \\
B(s)=(-\cos \theta,-\cos \theta \sin s,|\sin \theta|),
\end{array}\right.
$$

with $\gamma(0)=\left(x_{0}, y_{0}-\sin \theta, z_{0}\right)$ as initial point. Also, $k(s)=|\sin \theta|$ and $\tau(s)=\cos \theta$. So the unit circle $\mathbb{S}^{1}\left(x_{0}=y_{0}=0\right)$ and its parallels (that is, for every $\left.z_{0} \in \mathbb{R}\right)$ are Legendre curves in $\mathbb{E}^{3}$, as is easy to see. The Lancret invariant (4.3) becomes $\operatorname{Lancret}(\gamma)=\tau / k$ and we recover the Lancret expression (1.1) from the Introduction. Let us point out that since $f^{\prime} \equiv 0$ the second and third conditions (3.3) do not work.

With the Matlab file:

$>t=-20: 0 \cdot 1: 20$;

$>\operatorname{plot} 3(\sin (t) / \operatorname{sqrt}(2),-\cos (t) / \operatorname{sqrt}(2), t / \operatorname{sqrt}(2))$

$>\operatorname{plot} 3(\sin (t) / \operatorname{sqrt}(2),-\cos (t) / \operatorname{sqrt}(2),-t / \operatorname{sqrt}(2))$

we obtain Figures 1 and 2, which prove that $\gamma$ belongs to the cylinder

$$
\left(x-x_{0}\right)^{2}+\left(y-y_{0}\right)^{2}=\sin ^{2} \theta .
$$

According to Proposition 4.5, all slant curves in $\mathbb{E}^{3}$ with an affine $\alpha$, that is, $\alpha=$ $a s+b$ where $a \neq 0$, have a proper mean curvature vector field with the corresponding $\lambda=a^{2}$.

ExAmple 5.2. In the following we give an example of a nongeodesic $\theta$-slant curve in an exponential Euclidean warped product with $\beta>0$. Let us point out firstly that the 


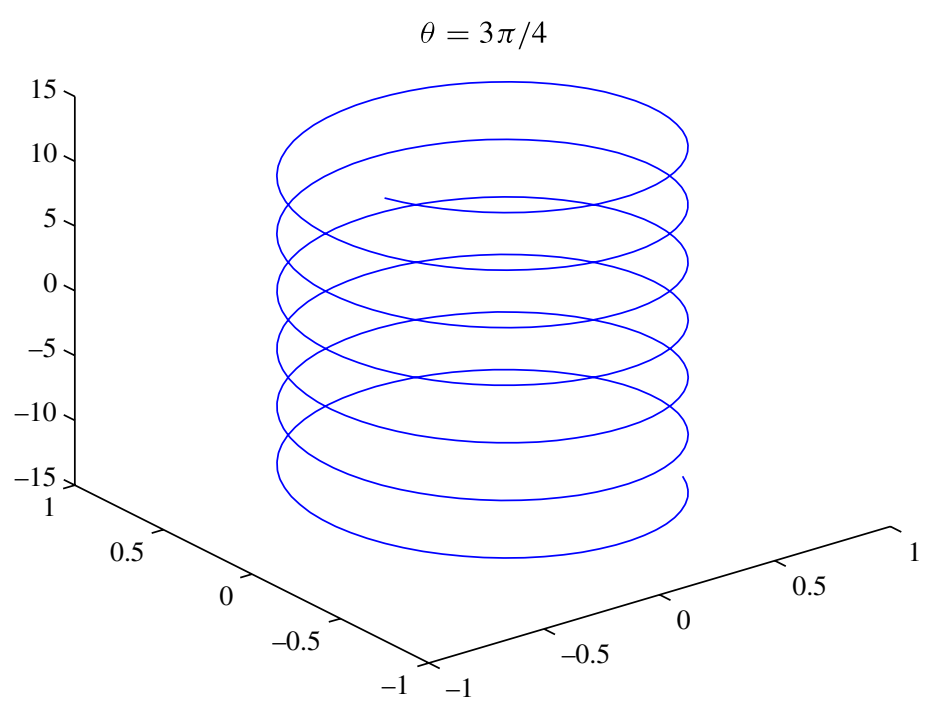

Figure 2. The second curve.

manifold $\mathbb{E} \times \exp z \mathbb{E}^{2}$ has constant sectional curvature $c=-1$, and by changing the $z$ coordinate one can obtain the upper half space model of the hyperbolic 3-space. More precisely, considering as in $[13, \mathrm{p}$. 46] the new coordinate $\tilde{z}=\exp (-z)$, we see that $\left(\mathbb{E} \times \exp z \mathbb{E}^{2}, g\right)$ is isometric to $\left(\mathbb{H}_{+}^{3}, g_{-1}\right)$, where $\mathbb{H}_{+}^{3}=\left\{(x, y, \tilde{z}) \in \mathbb{R}^{3} ; \tilde{z}>0\right\}$ and

$$
g_{-1}=\frac{1}{\tilde{z}^{2}}\left(d x^{2}+d y^{2}+d \tilde{z}^{2}\right) \text {. }
$$

Secondly, the $\theta$-slant curve

$$
\gamma(s)=\left(\frac{\sin \theta \exp (-\beta s \cos \theta)}{1+\beta^{2} \cos ^{2} \theta}(\sin s-\beta \cos \theta \cos s,-\cos s-\beta \cos \theta \sin s), s \cos \theta\right)
$$

corresponds to $\alpha(s)=s$ and has the tangent vector field

$$
T(s)=(\sin \theta \cos s \exp (-\beta s \cos \theta), \sin \theta \sin s \exp (-\beta s \cos \theta), \cos \theta)
$$

and the initial point $\gamma(0)=\left(\sin \theta /\left(1+\beta^{2}\right)\right)(-\beta \cos \theta,-1,0)$. Since

$$
\left\{E_{1}=\exp (-\beta z) \frac{\partial}{\partial x}, E_{2}=\exp (-\beta z) \frac{\partial}{\partial y}, E_{3}=\frac{\partial}{\partial z}\right\}
$$

is a $g$-orthonormal basis with

$$
\left[E_{1}, E_{2}\right]=0, \quad\left[E_{1}, E_{3}\right]=\beta E_{1}, \quad\left[E_{2}, E_{3}\right]=\beta E_{2},
$$

we derive the Levi-Civita connection:

$$
\nabla_{E_{1}} E_{1}=\nabla_{E_{2}} E_{2}=-\beta E_{3}, \quad \nabla_{E_{1}} E_{3}=\beta E_{1}, \quad \nabla_{E_{2}} E_{3}=\beta E_{2},
$$


the rest being zero. As a result,

$$
\begin{aligned}
\nabla_{\gamma^{\prime}} \gamma^{\prime}= & (\beta \sin \theta \cos \theta \cos s-\sin \theta \sin s) E_{1}+(\beta \sin \theta \cos \theta \sin s+\sin \theta \cos s) E_{2} \\
& -\beta \sin ^{2} \theta E_{3} .
\end{aligned}
$$

and then $k=\sqrt{1+\beta^{2}}|\sin \theta|$ in correspondence with (4.2a). Then we have two cases.

(i) $\theta \in(0, \pi)$. We get $k=\sqrt{1+\beta^{2}} \sin \theta$ and

$$
N(s)=\frac{1}{\sqrt{1+\beta^{2}}}\left((\beta \cos \theta \cos s-\sin s) E_{1}+(\beta \cos \theta \sin s+\cos s) E_{2}+(-\beta \sin \theta) E_{3}\right) .
$$

(ii) $\theta \in(\pi, 2 \pi)$. We have $k=-\sqrt{1+\beta^{2}} \sin \theta$ and

$$
N(s)=\frac{1}{\sqrt{1+\beta^{2}}}\left((-\beta \cos \theta \cos s+\sin s) E_{1}+(-\beta \cos \theta \sin s-\cos s) E_{2}+\beta \sin \theta E_{3}\right) .
$$

Let us remark that condition (3.3) reads $|\sin \theta| \leq \min \left\{1, \sqrt{1+\beta^{2}}|\sin \theta| /|\beta|\right\}$, which is true. For both cases we derive that $\tau=\cos \theta$ and we recover the Lancret invariant (4.3), $\operatorname{Lancret}(\gamma)=(\tau / k) \sqrt{1+\beta^{2}}$. From (3.4) we have the expressions

$$
B(s)=\frac{1}{\sqrt{1+\beta^{2}}}\left((-\cos \theta \cos s-\beta \sin s) E_{1}+(-\cos \theta \sin s+\beta \cos s) E_{2}+\sin \theta E_{3}\right)
$$

for case (i), and

$$
B(s)=\frac{1}{\sqrt{1+\beta^{2}}}\left((\cos \theta \cos s+\beta \sin s) E_{1}+(\cos \theta \sin s-\beta \cos s) E_{2}-\sin \theta E_{3}\right)
$$

for case (ii). In conclusion, $\gamma$ is a helix of order three and for $\beta=0$ we recover the first example with $x_{0}=y_{0}=z_{0}=0$. For $\theta=\pi / 2$ we obtain the Legendre curve $\gamma(s)=(\sin s,-\cos s, 0)$ which is the unit circle $\mathbb{S}^{1}$. Also, we can apply Proposition 4.5 and then $\gamma$ is a curve with proper mean curvature vector field and

$$
\lambda=k^{2}+\tau^{2}=1+\beta^{2} \sin ^{2} \theta .
$$

Since $\beta$ and $\theta$ are constants, $N$ is an eigenvector for the Laplacian with eigenvalue (5.2).

The Lancret theorem for the hyperbolic space in [2, p. 1506] states that a curve $\gamma$ in $\mathbb{H}_{+}^{3}$ is a general helix if and only if either:

(1) $\tau \equiv 0$ and $\gamma$ is a curve in some hyperbolic plane $\mathbb{H}^{2}(-1)$; or

(2) $\quad \gamma$ is in $\mathbb{H}_{+}^{3}$ with constant curvature and torsion.

Our curve (5.2) belongs to case (2). Recall that a general helix means, after [2], the existence of a Killing vector field $V$ with constant length along $\gamma$ making a constant angle with $T$. From the last line of Section 2 above we have that, for our case $\beta=1$,

$$
V(s)=\cos \varphi T(s)+\sin \varphi B(s)
$$


Case (i) with $\beta=1$ and $\theta=\pi / 4$

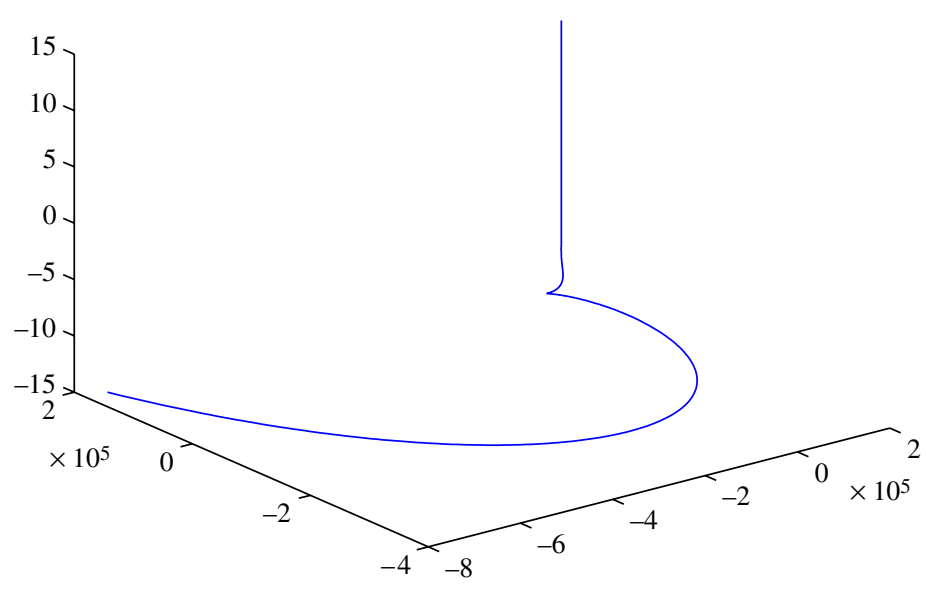

Figure 3. The first curve.

with

$$
\cot \varphi=\frac{\cos ^{2} \theta+1}{\sqrt{2}|\sin \theta| \cos \theta}
$$

We get

$$
\begin{aligned}
V(s)= & \frac{1}{\sqrt{1+\left(3+\sin ^{2} \theta\right) \cos ^{2} \theta}} \\
& \quad \times\left(e^{-s \cos \theta}|\sin \theta|(\cos s-\cos \theta \sin s, \sin s+\cos \theta \cos s), 2 \cos \theta\right) .
\end{aligned}
$$

Our curve (5.2) is plotted in Matlab with:

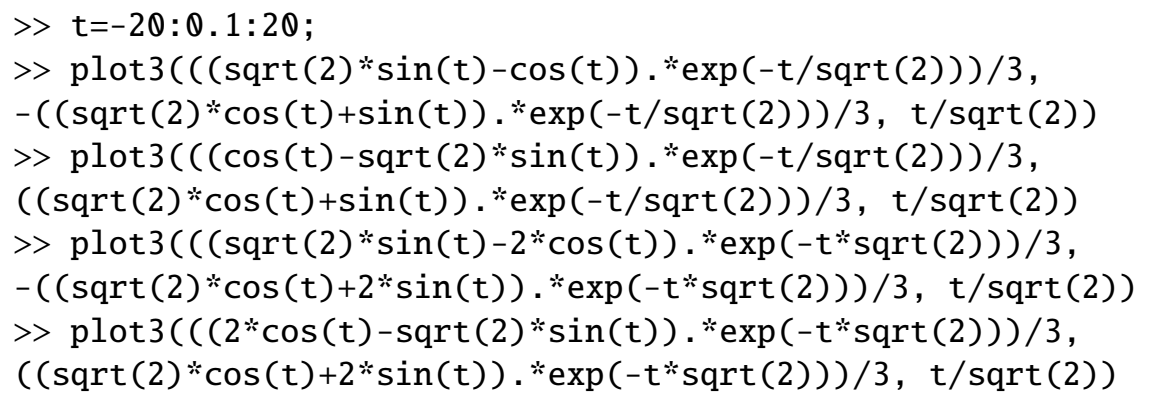

\section{Applications}

It is well known that some standard space-time models of the universe are (Lorentzian) warped products: Robertson and Walker, Schwarzschild, and others [18]. 
Case (ii) with $\beta=1$ and $\theta=3 \pi / 4$

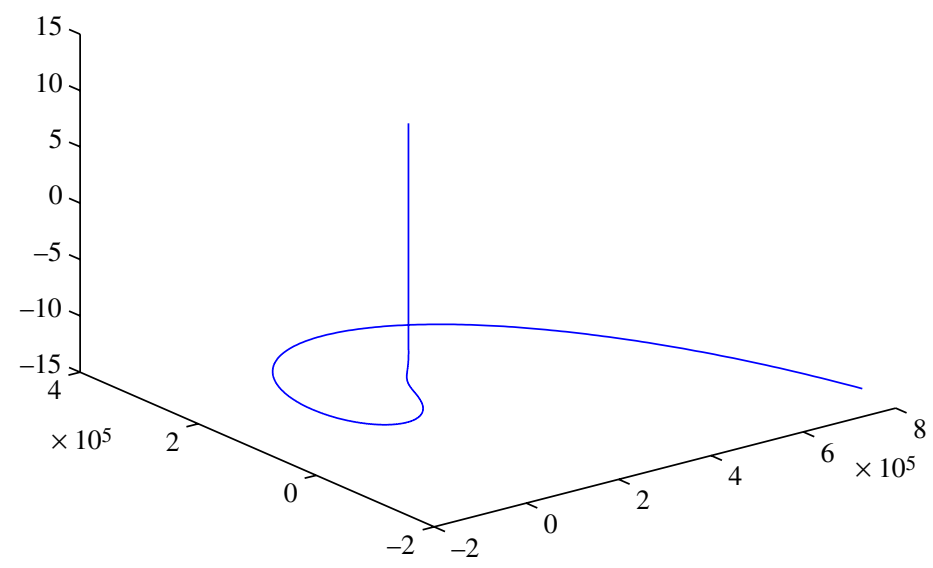

Figure 4. The second curve.

Case (i) with $\beta=2$ and $\theta=\pi / 4$

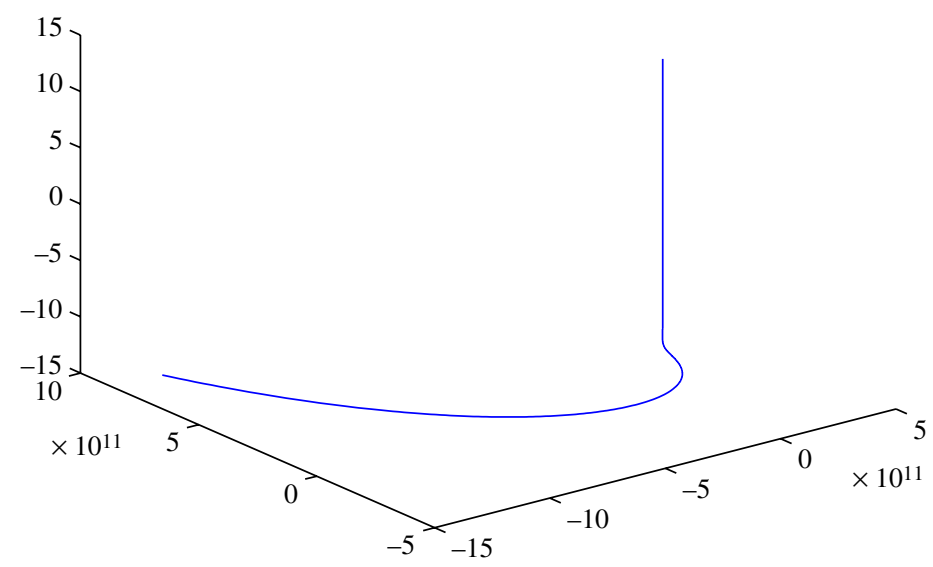

Figure 5. The third curve.

So, inspired also by the title of [14], we designate as a slant particle in the 3-manifold $M_{3}=I \times_{f} N_{2}$, a particle having as trajectory (or world-line) a slant curve. The present work characterises these particles from the point of view of their curvature and torsion and gives the Lancret expression (4.3) as first integral of motion.

Two interesting open problems arise naturally:

(1) A type of inverse problem; or more precisely, a dynamical problem: Does there exist a Lagrangian $L=L(x, y, z, \dot{x}, \dot{y}, \dot{z})$ on $M_{3}$ such that the slant curves are the solutions of the Euler-Lagrange equations for $L$ ? 
Case (ii) with $\beta=2$ and $\theta=3 \pi / 4$

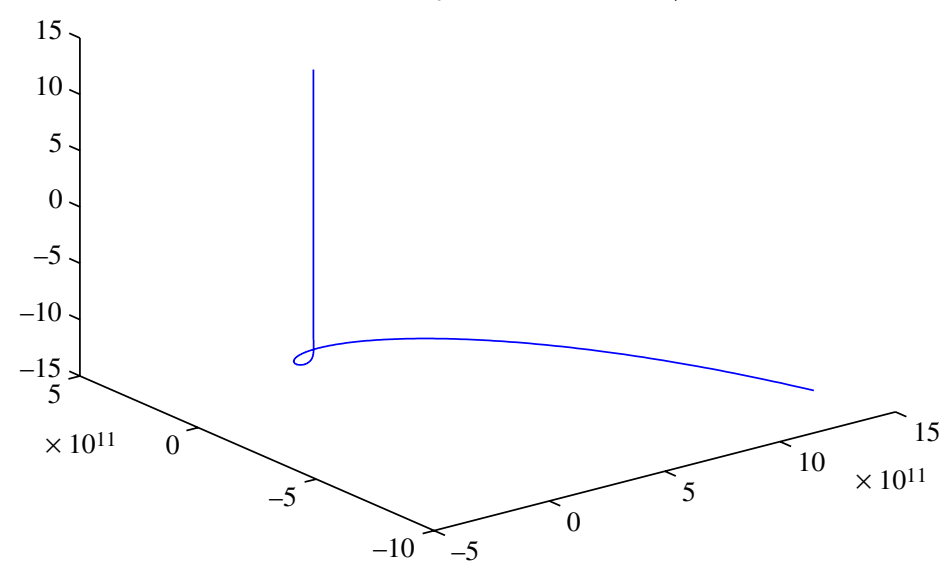

FIGURE 6. The fourth curve.

(2) A geometrical problem but related to (1): Does there exist a Riemannian (or Finsler) metric on $M_{3}$ having the slant curves as geodesics?

For the Euclidean case (5.1) the second-order equations are

$$
\left\{\begin{array}{l}
\frac{d^{2} x}{d s^{2}}=-x \\
\frac{d^{2} y}{d s^{2}}=-y \\
\frac{d^{2} z}{d s^{2}}=0
\end{array}\right.
$$

which correspond to the Lagrangian

$$
L=\frac{1}{2}\left(\dot{x}^{2}+\dot{y}^{2}+\dot{z}^{2}\right)-\frac{1}{2}\left(x^{2}+y^{2}\right) .
$$

For the exponential case (5.2) we derive the equations

$$
\left\{\begin{array}{l}
\frac{d^{2} x}{d s^{2}}=-\sin ^{2} \theta x+2 \cos \theta y \\
\frac{d^{2} y}{d s^{2}}=-2 \cos \theta x-\sin ^{2} \theta y \\
\frac{d^{2} z}{d s^{2}}=0
\end{array}\right.
$$

with the corresponding Lagrangian

$$
L=\dot{x} \dot{y}+\frac{\dot{z}^{2}}{2}+\cos \theta\left(y^{2}-x^{2}\right)-\sin ^{2} \theta x y .
$$


The general second-order equations corresponding to (4.1) are

$$
\left\{\begin{array}{l}
\frac{d^{2} x}{d s^{2}}=-\dot{y}-F^{\prime}(z) \dot{x} \dot{z} \\
\frac{d^{2} y}{d s^{2}}=\dot{x}-F^{\prime}(z) \dot{y} \dot{z} \\
\frac{d^{2} z}{d s^{2}}=0
\end{array}\right.
$$

for which we derive two first integrals,

$$
\left\{\begin{array}{l}
I_{1}=\frac{\dot{x}^{2}+\dot{y}^{2}}{f^{2}(z)}=\sin ^{2} \theta, \\
I_{2}=\dot{z}=\cos \theta .
\end{array}\right.
$$

Then the Lancret invariant (4.3) is a combination of these first integrals,

$$
\operatorname{Lancret}(\gamma)=\frac{\cos \theta}{|\sin \theta|}=\frac{f(z) \dot{z}}{\sqrt{(\dot{x})^{2}+(\dot{y})^{2}}}
$$

\section{Acknowledgement}

We are extremely grateful to our colleague Marian Ioan Munteanu with whom we collaborated on [8]. He inspired us to obtain the main results of the present paper.

\section{References}

[1] Ch. Baikoussis and D. E. Blair, 'On Legendre curves in contact 3-manifolds', Geom. Dedicata 49(2) (1994), 135-142.

[2] M. Barros, 'General helices and a theorem of Lancret', Proc. Amer. Math. Soc. 125(5) (1997), 1503-1509.

[3] M. Belkhelfa, I.-E. Hirica, R. Rosca and L. Verstraelen, 'On Legendre curves in Riemannian and Lorentzian Sasaki spaces', Soochow J. Math. 28(1) (2002), 81-91.

[4] R. L. Bishop and B. O'Neill, 'Manifolds of negative curvature', Trans. Amer. Math. Soc. 145 (1969), 1-49.

[5] D. E. Blair, Riemannian Geometry of Contact and Symplectic Manifolds, 2nd edn. Progress in Mathematics, 203 (Birkhäuser, Boston, MA, 2010).

[6] D. E. Blair, F. Dillen, L. Verstraelen and L. Vrancken, 'Deformations of Legendre curves', Note Mat. 15(1) (1995), 99-110 (1997).

[7] C. Călin and M. Crasmareanu, 'Slant curves in three-dimensional normal almost contact geometry', Mediterr. J. Math., to appear; doi:10.1007/s00009-012-0217-1.

[8] C. Călin, M. Crasmareanu and M. I. Munteanu, 'Slant curves in three-dimensional $f$-Kenmotsu manifolds', J. Math. Anal. Appl. 394(1) (2012), 400-407.

[9] C. Camci, Y. Yayli and H. H. Hacisalihoglu, 'On the characterization of spherical curves in threedimensional Sasakian spaces', J. Math. Anal. Appl. 342(2) (2008), 1151-1159.

[10] J. T. Cho, J.-I. Inoguchi and J.-E. Lee, 'On slant curves in Sasakian 3-manifolds', Bull. Aust. Math. Soc. 74(3) (2006), 359-367.

[11] J. T. Cho and J.-E. Lee, 'Slant curves in contact pseudo-Hermitian 3-manifolds', Bull. Aust. Math. Soc. 78(3) (2008), 383-396. 
[12] Ü. Çiftçi, 'A generalization of Lancret's theorem', J. Geom. Phys. 59(12) (2009), 1597-1603.

[13] F. Dillen, M. I. Munteanu, J. Van der Veken and L. Vrancken, 'Classification of constant angle surfaces in a warped product', Balkan J. Geom. Appl. 16(2) (2011), 35-47.

[14] A. Ferrández, J. Guerrero, M. A. Javaloyes and P. Lucas, 'Particles with curvature and torsion in three-dimensional pseudo-Riemannian space forms', J. Geom. Phys. 56(9) (2006), 1666-1687.

[15] D. Fetcu, 'Biharmonic Legendre curves in Sasakian space forms', J. Korean Math. Soc. 45(2) (2008), 393-404.

[16] S. Izumiya and N. Takeuchi, 'New special curves and developable surfaces', Turkish J. Math. 28(2) (2004), 153-163.

[17] J.-E. Lee, 'On Legendre curves in contact pseudo-Hermitian 3-manifolds', Bull. Aust. Math. Soc. 81(1) (2010), 156-164.

[18] B. O’Neill, Semi-Riemannian Geometry. With Applications to Relativity, Pure and Applied Mathematics, 103 (Academic Press, New York, 1983).

[19] K. Smoczyk, 'Closed Legendre geodesics in Sasaki manifolds', New York J. Math. 9 (2003), 23-47 (electronic).

[20] M. M. Tripathi, 'A note on certain Legendre curves in a Kenmotsu manifold', Ganita 51(1) (2000), $57-58$.

[21] J. Welyczko, 'On Legendre curves in three-dimensional normal almost contact metric manifolds', Soochow J. Math. 33(4) (2007), 929-937.

[22] J. Welyczko, 'On Legendre curves in 3-dimensional normal almost paracontact metric manifolds', Results Math. 54(3-4) (2009), 377-387.

CONSTANTIN CĂLIN, Department of Mathematics,

Technical University 'Gh. Asachi', 700049 Iaşi, Romania

e-mail:c0nstc@yahoo.com

MIRCEA CRASMAREANU, Faculty of Mathematics, University 'Al. I. Cuza', 700506 Iaşi, Romania

e-mail: mcrasm@uaic.ro 